\title{
O Regime de Metas de Inflação do Brasil é Crível?*
}

\author{
Vladimir Kühl Teles ${ }^{* *}$ \\ Joana Nemoto ${ }^{* * *}$
}

Sumário: 1. Introdução; 2. Fundamentação teórica; 3. A análise empírica; 4. Conclusões e implicações políticas.

Palavras-chave: credibilidade; metas de inflação; política monetária.

Códigos JEL: E52; E58.

Um Banco Central que estabelece e mantém uma meta de inflação baixa e estável, e abdica-se do uso do trade-off entre produto real e inflação como instrumento de política é definido como crível. Um modelo de série de tempo foi estimado com o objetivo de verificar a credibilidade do regime de metas de inflação brasileiro. Ao mesmo tempo, um índice de credibilidade da política monetária foi construído a partir de um modelo estado-espaço. Em tal modelo a utilização do filtro de Kalman sob o trade-off entre inflação e produto indica a evolução da credibilidade do Banco Central brasileiro ao combater a inflação.

A Central Bank that achieves and maintains its objective of a low and stable inflation rate, and does not attempt to exploit the tradeoff between real output and inflation is defined to be credible. A time series model was estimated in order to verify the brazilian inflation target regime's credibility. At the same time, a monetary policy's credibility index was built upon a State-Space model. In this model a random coeficients Kalman filter model of the inflation-output trade-off indicates the evolution in the credibility of the brazilian Central Bank as an inflation fighter.

\section{Introdução}

Após mais de uma década da implementação do regime de metas de inflação como um regime monetário "pioneiro" por Nova Zelândia, Chile, Austrália e Ca-

\footnotetext{
${ }^{*}$ Artigo recebido em nov. 2003 e aprovado em mar. 2005. Os autores agradecem os comentários valiosos realizados por Joaquim P. Andrade, Adolfo Sachsida, Benjamin Tabak e Maria Eduarda A. Tannuri. Todos os erros são de nossa inteira responsabilidade.

${ }^{* *}$ Fundação Getúlio Vargas, Escola de Economia de São Paulo (EESP/FGV). E-mail: vkteles@fgvsp.br

${ }^{* * *}$ Universidade de Brasília - UnB, Departamento de Economia. E-mail: jonemoto@unb.br
} 
nadá, mais de 20 países vieram a adotar tal regime monetário, ${ }^{1}$ sendo este considerado por muitos como um regime monetário ideal. Nesse sentido, a popularidade deste mecanismo de política tem aumentado, tanto pelo aumento do número de países que o adotam como pelo número crescente de economistas que o indicam como principal meio de manter a inflação em um nível baixo e estável, como, por exemplo, Taylor, J., Fischer, S., Freedman, C. e King, M. (ver Federal Reserve Bank of Kansas City (1996)).

Nesse contexto, ao descrever as vantagens de tal regime monetário, os seus adeptos enfatizam que um regime de metas de inflação pode apresentar uma maior transparência de objetivos de política monetária, que, por sua vez, pode implicar em um aumento da credibilidade da política monetária, o que é consideravelmente desejável para fins de performance macroeconômica. Assim, a principal questão envolvida na discussão da eficiência do regime de metas de inflação a fim de estabilizar a inflação a níveis baixos parece estar relacionada aos possíveis ganhos de credibilidade à política monetária que esta possa implicar.

Diante disso, o objetivo central do presente artigo é estimar a credibilidade da política monetária no Brasil para as últimas duas décadas, dando um enfoque prioritário às possíveis variações de credibilidade incorridas a partir da implementação do regime de metas de inflação em 1999. Para tanto é utilizada uma definição simples e familiar de credibilidade seguindo Razzak (2001), onde define-se que o Banco Central tem credibilidade se não explorar o trade-off de curto prazo entre inflação e produto. Nesse contexto, seguindo Lucas (1972, 1973), a utilização de tal relação só implica em algum resultado real se não for antecipada, logo a utilização de tal trade-off por si só seria um indicativo que o Banco Central teria se desviado dos seus alvos expostos aos agentes econômicos. Dessa forma, pode-se resumir tal definição de credibilidade de política como o grau de confiança que os agentes tem de que o trade-off entre inflação e produto no curto prazo não será usado.

Ao mesmo tempo, como demonstrado primeiramente por Lucas (1973), a magnitude do trade-off entre inflação e produto eleva-se quando este não é utilizado pela autoridade monetária. Logo, a mensuração da própria magnitude de tal trade-off vem a ser uma medida plausível para a não exploração de tal relação, e, como conseqüência imediata, para a credibilidade de política monetária. Nesse sentido, tal relação entre o uso do trade-off entre inflação e produto e a sua magnitude é corroborada pela tradição Novo Keynesiana embora sob uma interpretação

\footnotetext{
${ }^{1}$ Dentre os quais destacam-se, por exemplo, Brasil, Reino Unido, Finlândia, Suécia, México, Israel, Espanha, Coréia, Peru, Colômbia, África do Sul, Rep. Checa, Suiça, Noruega, Hungria, Tailândia, Islândia e Polônia
} 
causal distinta, como pode ser observado em Ball et alii (1988). Sendo assim, independentemente da tradição a ser seguida (Novo Clássica ou Novo Keynesiana) a mensuração de credibilidade aqui utilizada mantêm-se teoricamente aceitável.

Sob um ponto de vista metodológico busca-se aqui conduzir estimações do modelo de Lucas (1973) para o Brasil para o período 1980.1-2002.8, com dados mensais, obtendo uma medida para o trade-off entre produto e inflação. Nesse sentido, são inseridas ao modelo dummies iterativas para captar as variações de credibilidade em dois momentos específicos do tempo, o Plano Real, em 1994, e a adoção do regime de metas de inflação, em 1999. Por fim, uma série mensal para credibilidade de política monetária é construída a partir da condução do filtro de Kalman à regressão estimada, de forma a ser possível verificar pontualmente as variações de credibilidade no período considerado.

Assim, o presente trabalho se divide da forma como se segue: No próximo tópico é realizada uma breve descrição do modelo a ser estimado, referente a Lucas (1973). O terceiro tópico apresenta as estimações e apresentação dos resultados. $\mathrm{O}$ último tópico reserva-se às considerações finais.

\section{Fundamentação Teórica}

\subsection{Medidas de credibilidade}

Esta subseção busca descrever brevemente a base teórica para a construção de uma medida de credibilidade da política monetária. Nesse sentido discute-se as diversas metodologias que visam tal mensuração, explicar por que a metodologia aqui escolhia foi considerada apropriada e explicar sucintamente a sua fundamentação.

Nesse sentido, a literatura econômica tem se dado conta da importância da credibilidade da política monetária como fundamental para a eficácia de tais políticas a partir de Sargent (1982), que, ao considerar quatro episódios de processos de desinflação, concluiu que a credibilidade da política monetária figura como fator chave em tais processos. Nesse sentido, se os agentes racionais consideram a política de desinflação do Banco Central com credibilidade, então as expectativas de inflação baixa irão implicar rapidamente no comportamento de escolha de preço dos agentes, produzindo uma inflação menor sem reduzir, entretanto, o nível de atividade econômica. Dessa forma, os custos de uma política de inflação baixa dependem crucialmente da credibilidade de tal política.

Entretanto, resultados mais palpáveis da relação entre credibilidade e eficácia da política monetária dependem da construção de uma medida de credibilidade. Nesse sentido, a primeira tentativa de construir tal medida remete a Blanchard 
(1984) e Perry (1983) que utilizaram os erros de previsão de uma curva de Phillips estimada como um indicador de credibilidade. Assim, a idéia por trás de tal indicador é relacionar credibilidade a previsibilidade.

Uma outra alternativa para medir credibilidade de um processo de combate à inflação, sugerida originalmente por Sargent (1983) é a velocidade de desinflação. Nesse sentido, Ball $(1994,1995)$ alicerça um modelo teórico onde fica evidenciado que quanto maior a velocidade de desinflação, menor os custos incorridos nesta política, sendo possível interpretar que a medida de velocidade de desinflação como medida consistente de credibilidade.

Em seqüencia, Cukierman (1986) sugere que a falta de credibilidade do Banco Central advém da impossibilidade deste não controlar a taxa de crescimento da moeda perfeitamente, de forma que ocorre a tendência da autoridade monetária extrapolar a oferta de moeda, implicando em uma inflação adicional não esperada, e em conseqüência, na perda de credibilidade.

Um importante avanço metodológico na mensuração da credibilidade da política monetária advém por sua vez de Hardouvelis e Barnhart (1989), onde os efeitos nos preços de anúncios semanais de variações no M1 são estimados a partir da utilização do filtro de Kalman, construindo-se uma série de credibilidade da política monetária para o período 1978-1984 para os Estados Unidos. A partir de então, a utilização do filtro de Kalman torna-se uma prerrogativa extremamente útil em modelos com credibilidade variável no tempo, como, por exemplo, Faust e Svensson (1998). Por fim, formulando uma medida alternativa, Blinder (1999) constrói índices de credibilidade do Banco Central através da aplicação de questionários a diversos agentes econômicos.

No que se refere precisamente à credibilidade das metas de inflação, Svensson (1993) sugere um teste para a credibilidade das metas, onde é construído um intervalo consistente dos retornos reais dos títulos nominais. Dessa forma, basta verificar se a taxa de juros real ou os retornos reais esperados ficam situados fora do intervalo para que se possa rejeitar a hipótese de credibilidade. O autor faz o teste para Nova Zelândia, Canadá e Suécia. Enquanto os testes para o Canadá são inconclusivos, não se pode rejeitar credibilidade para a Nova Zelândia após a metade de 1992 e rejeita-se credibilidade para a Suécia. Outra importante conclusão é a de que pode haver algum tempo para o ganho de credibilidade logo após o anúncio das metas inflacionárias.

Ao mesmo tempo, a fim de realizar um teste de credibilidade para diversos regimes de metas de inflação, Razzak (2001) inspira-se em Lucas (1973) e Faust e Svensson (1998) ao relacionar credibilidade da política monetária ao trade-off entre inflação e produto. Nesse sentido, considerando-se que os agentes tem custos 
de ajustar preços e salários, ${ }^{2}$ o trade-off entre produto e inflação tende a elevarse quando os agentes não esperam choques monetários. Como conseqüência a ocorrência de tais choques tendem a diminuir a magnitude do trade-off. Sendo assim, a magnitude do trade-off está intimamente relacionada com a expectativa da não existência de choques monetários, ou, em outras palavras, com a credibilidade da política monetária de inflação baixa. Ao conduzir tal teste, Razzak (2001) observou que, em determinados países como Austrália, Nova Zelândia, e Suécia, o regime de metas de inflação mostrou-se crível, enquanto que no Canadá, e no Reino Unido, o resultado mostrou-se menos óbvio, uma vez que a constatação da credibilidade do regime de metas de inflação não pode ser estatisticamente confirmado.

Assim, diante de tantas alternativas de mensuração da credibilidade da política monetária qual é a mais adequada para a estimação a ser realizada aqui? Sob tal questão, o presente trabalho buscou seguir dois critérios de avaliação das estimações de credibilidade de política monetária. O primeiro refere-se a generalidade do índice a ser utilizado. Nesse sentido, cabe notar que embora uma previsibilidade perfeita implique em credibilidade da política monetária, erros de previsão não implicam, necessariamente, em perda de credibilidade, uma vez que tais erros podem ter sido causados por choques exógenos à política monetária. Assim, em tal contexto, a noção de credibilidade engloba a noção de previsibilidade, o que nos leva a descartar índices como os de Blanchard (1984) e Perry (1983). Utilizando o critério de generalidade o indicador de Sargent (1983) também parece não ser adequado para o estudo aqui proposto, uma vez que tem por objeto de estudo exclusivo a credibilidade da política monetária em períodos de desinflação.

O segundo critério a ser considerado é a necessidade de se construir um índice temporalmente variável de credibilidade, uma vez que variações na política implicam em mudanças de credibilidade, como demonstrado por Faust e Svensson (1998). Tal critério descarta os indicadores oriundos de Cukierman (1986), Svensson (1993) e Blinder (1999).

Assim, diante da necessidade do presente trabalho de se construir uma série de credibilidade que mantenha a generalidade de tal conceito, o indicador considerado mais apropriado para os fins do presente artigo remete-se a Razzak (2001). Nesse caso, embora tal indicador não seja construído na forma de série temporal, é possível adaptá-lo à metodologia utilizada por Hardouvelis e Barnhart (1989), ao

\footnotetext{
${ }^{2}$ Em uma abordagem novo-clássica tais custos advém dos custos de obtenção e processamento de informação, como em Lucas $(1972,1973)$, enquanto que em uma abordagem novo keynesiana tais custos são relacionados a um determinado grau de rigidez nominal existente na economia, como em Mankiw (1985)
} 
conduzir-se o filtro de Kalman à medida de credibilidade de Razzak. A idéia por trás do indicador de credibilidade de Razzak (2001) é que a autoridade monetária tem credibilidade se não surpreender os agentes econômicos com um choque monetário. Nesse sentido, tal "surpresa" teria um efeito negativo imediato sobre o trade-off entre inflação-produto, como predito por Lucas (1973) e amplamente reconhecido na teoria econômica. Dessa forma, a estimação apropriada do tradeoff entre inflação-produto implica em uma boa medida de credibilidade da política monetária. A estimação apropriada de tal relação é construída com base em Lucas (1973), cujo modelo é resumido na seção a seguir.

\subsection{O Modelo Teórico}

Esta seção busca descrever brevemente o modelo teórico por trás da estimação do trade-off entre inflação-produto, seguindo Lucas (1973). Tal modelo é construído a partir da curva de oferta agregada de Lucas (1973), que será combinada a uma curva de demanda para edificar as equações de equilíbrio a serem estimadas para inferirem o trade-off entre produto e inflação.

Nesse sentido, o modelo de Lucas (1973) representa uma análise estrutural de tal trade-off inserida em um paradigma construído inicialmente por Friedman (1968) e Phelps (1968). Tal modelo é adequado para a análise aqui realizada por duas razões centrais. A primeira é a garantia dada pelo modelo da neutralidade de longo-prazo da moeda. A segunda razão se deve ao não questionamento da sua forma funcional por parte da escola novo-keynesiana, como extensamente argumentado por Ball et alii (1988). Nesse ponto, a escola novo-keynesiana discute algumas algumas interpretações construídas a partir desta relação funcional e não sua veracidade. ${ }^{3}$

Outro aspecto importante é que a curva de oferta de Lucas possui a hipótese da taxa natural, isto implica a não que não existe apenas uma única taxa de inflação condizente com a escolha do Banco Central de elevar o produto acima do produto normal permanentemente. A rigidez entra no modelo devido ao acesso dos ofertantes à informação imperfeita sobre o nível de preços geral corrente. ${ }^{4}$

Igualando-se as curvas de demanda e oferta agregada e resolvendo para os níveis de preços e produto reais, o autor deriva duas equações. A primeira (1) é a equação

\footnotetext{
${ }^{3}$ Em uma abordagem alternativa a curva de oferta de Calvo, referente a Calvo (1983), Rotemberg (1982) e Taylor (1980), baseada em uma arcabouço de contratos justapostos vai de encontro a curva de oferta de Lucas não resultando em uma não confirmação da hipótese de taxa natural.

${ }^{4}$ Nesse sentido, como já difundido na literatura, uma política monetária expansionista pode levar os produtores a enxergar um aumento no nível geral de preços como uma elevação apenas na demanda pelo seu produto, fazendo com que, no curto prazo, haja uma elevação do produto.
} 
do produto real ( $y_{c t}$, componente cíclico) em função da taxa de crescimento do produto nominal $\left(\Delta x_{t}\right)$ e do produto real defasado $\left(\Delta y_{c t-1}\right)$ mais um termo de erro $\left(u_{1, t}\right)$. O parâmetro $\varphi$ é usado para testar a hipótese do trade-off e da credibilidade. A equação (2) apresenta a taxa de variação dos preços em função de um parâmetro de tendência $(\delta)$, da taxa de crescimento dos produtos nominal atual e defasada, do hiato do produto real defasado, mais um termo de erro $\left(u_{2, t}\right) .^{5}$

$$
\begin{gathered}
y_{c, t}=-\alpha \varphi+\varphi \Delta x_{t}+\beta y_{c, t-1}+u_{1, t} \\
\Delta P_{t}=-\delta+(1-\varphi) \Delta x_{t}+\varphi \Delta x_{t-1}-\beta \Delta y_{c, t-1}+u_{2, t}
\end{gathered}
$$

onde o parâmetro de credibilidade pode ser escrito como $\varphi=\frac{\chi^{2} \gamma}{(1-\varphi)^{2} \sigma_{x}^{2}+\chi^{2}(1+\gamma)}$, sendo a resposta do componente cíclico do produto às variações nos preços relativos capturada por $\gamma$, a variância do desvio percentual do nível de preços em um dado mercado do nível geral de preços por $\chi^{2}$, e a variância na mudança da demanda agregada por $\sigma_{x}^{2}$.

Assim, nota-se que se a variância da mudança na demanda agregada tende a zero, o valor de $\varphi$ tende a $\frac{\gamma}{(1+\gamma)}$. Se a variância da mudança na demanda agregada tende ao infinito, $\varphi$ tende monotonicamente a zero, ou em outras palavras, o parâmetro de credibilidade é maior em um regime de inflação estável e com um Banco Central que não utiliza o trade-off, do que em alta inflação e com um banco central que explora este trade-off.

Dessa forma, a fim de se obter uma medida para credibilidade da política monetária deve-se conduzir as estimações das equações (1) e (2). Dessa forma, o presente trabalho visa estimar tais relações para o Brasil a fim de podermos discutir a credibilidade da política monetária brasileira. Entretanto, deve-se observar que as equações (1) e (2) não são mantidas quando há autocorrelação na taxa de crescimento do produto nominal, o que ocorre para o caso brasileiro. Assim, considerando que $E\left(x_{t} \mid I_{t}\right)=\delta+x_{t-1}+\sum_{i} \rho_{i} \Delta x_{t-i}$, ou seja, que $E\left(\Delta x_{t} \mid I_{t}\right)$ segue um processo autoregressivo dependendo dos períodos defasados $i$, as equações de equilíbrio supracitadas seriam dadas por:

$$
\begin{gathered}
y_{c, t}=-\eta \delta+\eta \Delta x_{t}+\phi y_{c, t-1}+\eta \sum_{i} \rho_{i} \Delta x_{t-i} \\
\Delta P_{t}=-\beta+(1-\eta) \Delta x_{t}+\eta \Delta x_{t-1}-\phi y_{c, t}+\eta \sum_{i} \rho_{i}\left(\Delta x_{t-i}-\Delta x_{t-i-1}\right)
\end{gathered}
$$

\footnotetext{
${ }^{5}$ Os termos de erro $\left(u_{1, t}\right)$ e $\left(u_{2, t}\right)$ são ruídos branco e não correlacionados
} 
Assim, podemos estender o modelo para excluir o efeito de uma possível autocorrelação nos choques ao adicionar termos defasados às equações de equilíbrio. (ver Lucas (1973) nota 5). Para o caso brasileiro as ordens de autocorrelação de $\Delta x$ foram calculadas e estão expressas na tabela 1 bem como os testes de autocorrelação dos resíduos o modelo (tabela 3), ou seja, os testes de Ljung-Box (Estatística Q) e de Breusch-Godfrey (Testes LM), evidenciando a ausência de correlação dos resíduos da especificação apresentada. Tais resultados nos deixam claro que para o caso brasileiro temos $i=1,2,10,11,12$ nas equações (3) e (4) acima.

Tabela 1

Autocorrelação serial de $\Delta x$

\begin{tabular}{|c|c|c|c|c|}
\hline Variável & Coeficiente & Erro Padrão & Estatística $t$ & Prob. \\
\hline $\mathrm{AR}(1)$ & 0.2909 & 0.0813 & 3.5740 & 0.0004 \\
\hline $\operatorname{AR}(2)$ & 0.4514 & 0.0664 & 6.7949 & 0.0000 \\
\hline $\operatorname{AR}(10)$ & -0.1143 & 0.0577 & -1.9783 & 0.0490 \\
\hline $\operatorname{AR}(11)$ & -0.1761 & 0.0500 & -3.5217 & 0.0005 \\
\hline $\operatorname{AR}(12)$ & 0.4936 & 0.0544 & 9.0668 & 0.0000 \\
\hline \multicolumn{5}{|c|}{ Testes de correlação serial dos resíduos: } \\
\hline Teste & Estatística & Prob. & & \\
\hline Estatística $Q$ (12 lags) & 7.3090 & 0.2930 & & \\
\hline Estatística $Q$ (24 lags) & 17.7880 & 0.4020 & & \\
\hline Teste $L M-F$ (12 lags) & 1.1211 & 0.3434 & & \\
\hline Teste $L M-N R^{2}$ (12 lags) & 12.8606 & 0.3792 & & \\
\hline Teste $L M-F(24$ lags $)$ & 0.9775 & 0.4903 & & \\
\hline Teste $L M-N R^{2}$ (24 lags) & 19.2383 & 0.5063 & & \\
\hline
\end{tabular}

\section{A Análise Empírica}

Os dados utilizados no presente estudo tem como fonte o IPEA e o Banco Central, onde a série de produto tem como fonte o PIB mensal divulgado pelo Banco Central e a série de preços o Índice Nacional de Preços ao Consumidor (INPC). Utilizou-se periodicidade mensal compreendendo o período 1980:1-2002:8, tendo as séries sido desazonalizadas a partir do método de media-movel multiplicativa, e, em seguida, para se obter o comportamento cíclico da série de produto real utilizou-se o filtro de Hodrick-Prescott (filtro HP).

Após a realização destes procedimentos os devidos testes de raiz unitária (tabela 2) foram conduzidos (Teste Phillips-Perron ${ }^{6}$ constando-se que, ao se definindo $\Delta x$ como a primeira diferença de $x$ e $\Delta P$ como a primeira diferença de $P$

\footnotetext{
${ }^{6}$ o teste de Phillips-Perron é mais adequado no presente caso que o teste ADF por controlar o efeito da correlação serial para o teste de raiz unitária.
} 
tornamos todas as séries estacionárias, uma vez que $y_{c}$ já é estacionária, de forma que a possibilidade de regressão espúria é descartada.

Tabela 2

Testes de raiz unitária

\begin{tabular}{lcc}
\hline & Estatística $t$ ajustada & Prob. \\
\hline$x$ & 0.1457 & 0.9976 \\
$P$ & 0.1261 & 0.9974 \\
$\Delta x$ & -6.4488 & 0.0000 \\
$\Delta P$ & -3.4566 & 0.0463 \\
\hline
\end{tabular}

A fim de testar as variações na credibilidade da política monetária ocorridas no Brasil diante das recentes mudanças no quadro macroeconômico brasileiro, e pelas exposições teóricas feitas até então, foram elaborados dois modelos econométricos com finalidades distintas. O primeiro objetiva o confronto entre a credibilidade da política monetária de três períodos fundamentais: o período pré-Plano Real, o período pós-Real até o estabelecimento do regime de metas de inflação e o período a partir de seu estabelecimento até o final da série. Os resultados de tais estimações são apresentados na subseção 3.1.

O segundo modelo, a ser apresentado na subseção 3.2., constitui-se na construção de um indicador de periodicidade mensal para a credibilidade da política monetária para o período compreendido na amostra. Nesse respeito utiliza-se um modelo estado-espaço através da condução do filtro de Kalman para parâmetro de credibilidade, aplicando a metodologia proposta por Hardouvelis e Barnhart (1989) ao modelo estimado na subseção 3.1.

\subsection{Os Resultados estimados}

A fim de testar as variações na credibilidade da política monetária ocorridas no Brasil diante das recentes mudanças no quadro macroeconômico brasileiro são inseridas dummies iterativas para o segundo e terceiro períodos (o período pósReal até o estabelecimento do regime de metas de inflação e o período que inicia-se a partir de seu estabelecimento até o final da série, respectivamente) ao se estimar as equações (3) e (4) apresentadas na seção anterior. A hipótese testada será então a mudança do parâmetro $\eta$ na passagem dos períodos relacionados. Dessa forma as dummies são definidas como,

$$
R E A L \begin{cases}1, & \text { para } t \text { entre 1994:08 e 1998:12 } \\ 0, & \text { caso contrário }\end{cases}
$$

$\mathrm{e}$ 


$$
\operatorname{IT} \begin{cases}1, & \text { para } t \text { entre 1999:01 e 2002:08 } \\ 0, & \text { caso contrário }\end{cases}
$$

Dessa forma, as estimações dos parâmetros $R E A L$ e $I T$ fornecem a magnitude do ganho de credibilidade com relação ao período pré-Plano Real, de forma que, se tais variáveis assumirem valores próximos chega-se à conclusão de rejeição do aumento de credibilidade da política monetária a partir da implementação do regime de metas de inflação, ou seja, a hipótese a ser testada é a igualdade entre dos valores estimados de $R E A L$ e $I T$.

Assim, a fim de se conduzir tais estimações recorreu-se à divisão da amostra em três alternativas, uma com a amostra inteira, outra utilizando-se dados a partir de 1990, e por fim, utilizando-se dados apenas para o período pós-Plano Real. Nesse último caso a dummie $R E A L$ é retirada para evitar a ocorrência de correlação perfeita entre regressores, de forma que, para esta amostra a hipótese a ser testada é simplesmente a significância do parâmetro $I T$.

Ao mesmo tempo, é natural supor que a estimação das equações (3) e (4) separadamente resulte em um problema de simultaneidade dada a provável relação ambígua de causalidade entre $y_{c t}$ e $\Delta x_{t}$. Nesse sentido, dois métodos de estimação são sugeridos. O primeiro, que não visa a correção da simultaneidade é a estimação das equações (3) e (4) de forma restrita. Nesse sentido, uma vez que estamos tratando de um sistema de equações, o método imediatamente sugerido é o de Mínimos Quadrados Iterativos (MQI), onde os coeficientes estimados são restritos pelas relações funcionais pré-dispostas. Nessa abordagem os coeficientes são obtidos a partir de uma construção da relação entre as estimações das duas equações que é conduzida seqüencialmente até obter-se a convergência a valores consistentes.

O segundo método de estimação busca solucionar o provável problema de simultaneidade do sistema. Para tanto, o método aqui sugerido é o FIML (FullInformation Maximum Likelihood ). Nesse sentido, uma vez que o modelo empírico é consagrado pela literatura econômica como bem especificado, este modelo apresenta ganhos de eficiência consideráveis com relação aos métodos de estimação com informação limitada, como dois estágios, GMM, ou LIML (Limited Information Maximum Likelihood). Ao mesmo tempo, uma vez que o método de estimação do FIML parte de uma estimação direta da máxima verossimilhança, ele também implica em ganhos de eficiência com relação a outros estimadores de informação completa como o de três estágios. Assim, os resultados das estimações são apresentados nas tabelas 3 a 9 . 
A tabela 3 apresenta os resultados estimados por Mínimos Quadrados Ordinários sem qualquer restrição aos coeficientes e com as equações sendo estimadas separadamente. Nestes resultados os coeficientes $\beta_{4}$ e $\beta_{5}$ que representam o aumento da credibilidade da política monetária nos período pós Plano Real e pós Metas Inflacionárias, respectivamente, não são significantes, de forma que tanto o Plano Real quanto a adoção do regime de metas de inflação não teriam refletido seus efeitos na trajetória da credibilidade da política monetária. Ao mesmo tempo um resultado interessante dessa estimação deve ser observado ao se comparar os coeficientes $\beta_{7}$ e $\beta_{8}$. Sob tal aspecto os valores de tais coeficientes são significantes e muito próximos, o que fornece uma evidência a favor da neutralidade da política monetária no longo prazo, que é um resultado imediato se $\left(1-\beta_{7}\right)+\beta_{8}=1$, ou seja, se $\beta_{7}=\beta_{8}$.

Tabela 3

Estimações do sistema (3)-(4) por MQO

\begin{tabular}{lcccc}
\hline Parâmetro & Coeficiente & Desv. Padrão & Estatística $t$ & Prob. \\
\hline$\beta_{1}$ & 0.0008 & 0.0020 & 0.4046 & 0.6859 \\
$\beta_{2}$ & 0.1424 & 0.0213 & 6.6741 & 0.0000 \\
$\beta_{3}$ & 0.6084 & 0.0482 & 12.6135 & 0.0000 \\
$\beta_{4}$ & 0.0447 & 0.0823 & 0.5436 & 0.5869 \\
$\beta_{5}$ & -0.0993 & 0.0944 & -1.0517 & 0.2934 \\
$\varphi_{1}$ & -0.1056 & 0.0210 & -5.0119 & 0.0000 \\
$\varphi_{2}$ & -0.0525 & 0.0199 & -2.6305 & 0.0088 \\
$\varphi_{10}$ & 0.0254 & 0.0196 & 1.2982 & 0.1948 \\
$\varphi_{11}$ & 0.0230 & 0.0201 & 1.1437 & 0.2532 \\
$\varphi_{12}$ & -0.0414 & 0.0213 & -1.9418 & 0.0527 \\
$\beta_{6}$ & 0.0019 & 0.0027 & 0.6990 & 0.4849 \\
$\beta_{7}$ & 0.3000 & 0.0309 & 9.7083 & 0.0000 \\
$\beta_{8}$ & 0.2640 & 0.0325 & 8.1227 & 0.0000 \\
$\beta_{9}$ & -0.0816 & 0.0796 & -1.0250 & 0.3059 \\
$\psi_{1}$ & 0.0267 & 0.0337 & 0.7919 & 0.4288 \\
$\psi_{2}$ & 0.0200 & 0.0268 & 0.7459 & 0.4561 \\
$\psi_{10}$ & 0.0756 & 0.0265 & 2.8562 & 0.0045 \\
$\psi_{11}$ & 0.2372 & 0.0303 & 7.8209 & 0.0000 \\
$\psi_{12}$ & 0.0400 & 0.0302 & 1.3238 & 0.1862 \\
\hline$y_{c, t}=\beta_{1}+\beta_{2} \Delta x_{t}+\beta_{3} y_{c, t-1}+\beta_{4}\left(R E A L * \Delta x_{t}\right)+\beta_{5}\left(I T * \Delta x_{t}\right)+\sum_{i} \varphi_{i} \Delta x_{t-i}$ \\
$\Delta P_{t}=\beta_{6}+\left(1-\beta_{7}\right) \Delta x_{t}+\beta_{8} \Delta x_{t-1}+\beta_{9} y_{c, t}+\sum_{i} \psi_{i}\left(\Delta x_{t-i}-\Delta x_{t-i-1}\right)$ \\
\hline
\end{tabular}


Ao se estimar o modelo restrito (por MQI) chegamos aos resultados apresentados na tabela 4. Nestes resultados podemos observar que tanto o período pós-Plano Real quanto o período a partir da adoção do regime de metas de inflação apresentaram valores para a credibilidade estimada superiores ao período como um todo. Entretanto a adoção do regime de metas de inflação não elevou significantemente a credibilidade da política monetária em comparação com o período pós-Real, o que pôde ser comprovado por um teste de hipótese da igualdade entre $\beta_{4}$ e $\beta_{5}$ (tabela 10). Ao repetir o exercício utilizando FIML (tabela 5) o Plano Real continua sendo um divisor de águas para a credibilidade da políica monetária, o que não permanece com o período de metas de inflação.

Tabela 4

Estimações do sistema (3)-(4) por MQI: 1980-2002

\begin{tabular}{lcccc}
\hline Parâmetro & Coeficiente & Erro Padrão & Estatística $t$ & Prob. \\
\hline$\delta \eta$ & -0.0064 & 0.0034 & -1.9175 & 0.0557 \\
$\eta$ & 0.1988 & 0.0236 & 8.4267 & 0.0000 \\
$\phi$ & 0.3069 & 0.0581 & 5.2823 & 0.0000 \\
$\beta_{4}$ & 0.3793 & 0.0715 & 5.3061 & 0.0000 \\
$\beta_{5}$ & 0.4286 & 0.0895 & 4.7903 & 0.0000 \\
$\rho_{1}$ & -0.4121 & 0.0897 & -4.5924 & 0.0000 \\
$\rho_{2}$ & -0.4650 & 0.0930 & -4.9998 & 0.0000 \\
$\rho_{10}$ & 0.0818 & 0.0980 & 0.8347 & 0.4043 \\
$\rho_{11}$ & 0.6491 & 0.1298 & 5.0010 & 0.0000 \\
$\rho_{12}$ & -0.6312 & 0.1208 & -5.2229 & 0.0000 \\
$\alpha$ & -0.0062 & 0.0023 & -2.7233 & 0.0067 \\
\multicolumn{5}{c}{} \\
\hline$y_{c, t}=-\eta \delta+\eta \Delta x_{t}+\phi y_{c, t-1}+\beta_{4}\left(R E A L * \Delta x_{t}\right)+\beta_{5}\left(I T * \Delta x_{t}\right)$ \\
$+\eta \sum_{i} \rho_{i} \Delta x_{t-i}$ \\
$\Delta P_{t}=-\alpha+(1-\eta) \Delta x_{t}+\eta \Delta x_{t-1}-\phi y_{c, t}+\left(1-\beta_{4}\right)\left(R E A L * \Delta x_{t}\right)$ \\
$+\beta_{4}\left(R E A L * \Delta x_{t-1}\right)+\left(1-\beta_{5}\right)\left(I T * \Delta x_{t}\right)+\beta_{5}\left(I T * \Delta x_{t-1}\right)$ \\
$+\eta \sum_{i} \rho_{i}\left(\Delta x_{t-i}-\Delta x_{t-i-1}\right)$ \\
\hline
\end{tabular}


Tabela 5

Estimações do sistema (3)-(4) por FIML: 1980-2002

\begin{tabular}{|c|c|c|c|c|}
\hline & Coeficiente & Erro Padrão & Estatística $z$ & Prob. \\
\hline$\delta \eta$ & -0.0014 & 0.0024 & -0.6131 & 0.5398 \\
\hline$\eta$ & 0.1790 & 0.0190 & 9.4189 & 0.0000 \\
\hline$\phi$ & 0.5421 & 0.0583 & 9.2934 & 0.0000 \\
\hline$\beta_{4}$ & 0.2151 & 0.0934 & 2.3016 & 0.0214 \\
\hline$\beta_{5}$ & 0.1101 & 0.1464 & 0.7519 & 0.4521 \\
\hline$\rho_{1}$ & -0.4583 & 0.0984 & -4.6555 & 0.0000 \\
\hline$\rho_{2}$ & -0.5161 & 0.1094 & -4.7195 & 0.0000 \\
\hline$\rho_{10}$ & 0.1843 & 0.0894 & 2.0610 & 0.0393 \\
\hline$\rho_{11}$ & 0.5111 & 0.0954 & 5.3552 & 0.0000 \\
\hline$\rho_{12}$ & -0.6852 & 0.0945 & -7.2484 & 0.0000 \\
\hline$\alpha$ & -0.0062 & 0.0038 & -1.6002 & 0.1095 \\
\hline \multicolumn{5}{|c|}{$\begin{array}{l}y_{c, t}=-\eta \delta+\eta \Delta x_{t}+\phi y_{c, t-1}+\beta_{4}\left(R E A L * \Delta x_{t}\right)+\beta_{5}\left(I T * \Delta x_{t}\right) \\
+\eta \sum_{i} \rho_{i} \Delta x_{t-i}\end{array}$} \\
\hline \multicolumn{5}{|c|}{$\begin{array}{l}\Delta P_{t}=-\alpha+(1-\eta) \Delta x_{t}+\eta \Delta x_{t-1}-\phi y_{c, t}+\left(1-\beta_{4}\right)\left(R E A L * \Delta x_{t}\right) \\
+\beta_{4}\left(R E A L * \Delta x_{t-1}\right)+\left(1-\beta_{5}\right)\left(I T * \Delta x_{t}\right)+\beta_{5}\left(I T * \Delta x_{t-1}\right) \\
+\eta \sum_{i} \rho_{i}\left(\Delta x_{t-i}-\Delta x_{t-i-1}\right)\end{array}$} \\
\hline
\end{tabular}

As tabelas 6 e 7 apresentam as mesmas estimações para o período que se inicia na década de 1990. Em ambos os casos $\beta_{4}$ e $\beta_{5}$ são significantemente diferentes de zero, e $\beta_{4}$ é maior que $\beta_{5}$. Porém os dois coeficientes não são significantemente diferentes (tabela 10). Assim, a conclusão básica de tais resultados é que o regime de metas de inflação manteve a credibilidade da política monetária alcançada pelo Plano Real. 
Tabela 6

Estimações do sistema (3)-(4) por MQI: 1990-2002

\begin{tabular}{|c|c|c|c|c|}
\hline & Coeficiente & Erro Padrão & Estatística $t$ & Prob. \\
\hline$\delta \eta$ & -0.0047 & 0.0044 & -1.0807 & 0.2807 \\
\hline$\eta$ & 0.1446 & 0.0325 & 4.4543 & 0.0000 \\
\hline$\phi$ & 0.2181 & 0.0848 & 2.5738 & 0.0106 \\
\hline$\beta_{4}$ & 0.5262 & 0.1169 & 4.5004 & 0.0000 \\
\hline$\beta_{5}$ & 0.4467 & 0.1251 & 3.5700 & 0.0004 \\
\hline$\rho_{1}$ & -0.4935 & 0.1674 & -2.9479 & 0.0035 \\
\hline$\rho_{2}$ & -0.6859 & 0.1966 & -3.4882 & 0.0006 \\
\hline$\rho_{10}$ & 0.2074 & 0.1928 & 1.0758 & 0.2829 \\
\hline$\rho_{11}$ & 0.8041 & 0.2641 & 3.0453 & 0.0025 \\
\hline$\rho_{12}$ & -0.8025 & 0.2558 & -3.1373 & 0.0019 \\
\hline$\alpha$ & -0.0096 & 0.0032 & -2.9641 & 0.0033 \\
\hline \multicolumn{5}{|c|}{$\begin{array}{l}y_{c, t}=-\eta \delta+\eta \Delta x_{t}+\phi y_{c, t-1}+\beta_{4}\left(R E A L * \Delta x_{t}\right)+\beta_{5}\left(I T * \Delta x_{t}\right) \\
+\eta \sum_{i} \rho_{i} \Delta x_{t-i}\end{array}$} \\
\hline \multicolumn{5}{|c|}{$\begin{array}{l}\Delta P_{t}=-\alpha+(1-\eta) \Delta x_{t}+\eta \Delta x_{t-1}-\phi y_{c, t}+\left(1-\beta_{4}\right)\left(R E A L * \Delta x_{t}\right) \\
+\beta_{4}\left(R E A L * \Delta x_{t-1}\right)+\left(1-\beta_{5}\right)\left(I T * \Delta x_{t}\right)+\beta_{5}\left(I T * \Delta x_{t-1}\right) \\
+\eta \sum_{i} \rho_{i}\left(\Delta x_{t-i}-\Delta x_{t-i-1}\right)\end{array}$} \\
\hline
\end{tabular}

Tabela 7

Estimações do sistema (3)-(4) por FIML: 1990-2002

\begin{tabular}{|c|c|c|c|c|}
\hline & Coeficiente & Erro Padrão & Estatística $z$ & Prob. \\
\hline$\delta \eta$ & -0.0013 & 0.0031 & -0.4350 & 0.6635 \\
\hline$\eta$ & 0.1259 & 0.0212 & 5.9292 & 0.0000 \\
\hline$\phi$ & 0.3748 & 0.0870 & 4.3064 & 0.0000 \\
\hline$\beta_{4}$ & 0.3606 & 0.0673 & 5.3596 & 0.0000 \\
\hline$\beta_{5}$ & 0.3090 & 0.1115 & 2.7711 & 0.0056 \\
\hline$\rho_{1}$ & -0.5794 & 0.2619 & -2.2119 & 0.0270 \\
\hline$\rho_{2}$ & -0.6673 & 0.3003 & -2.2224 & 0.0263 \\
\hline$\rho_{10}$ & 0.4642 & 0.2018 & 2.3008 & 0.0214 \\
\hline$\rho_{11}$ & 0.6852 & 0.2201 & 3.1131 & 0.0019 \\
\hline$\rho_{12}$ & -1.0321 & 0.2012 & -5.1288 & 0.0000 \\
\hline$\alpha$ & -0.0098 & 0.0056 & -1.7415 & 0.0816 \\
\hline \multicolumn{5}{|c|}{$\begin{array}{l}y_{c, t}=-\eta \delta+\eta \Delta x_{t}+\phi y_{c, t-1}+\beta_{4}\left(R E A L * \Delta x_{t}\right)+\beta_{5}\left(I T * \Delta x_{t}\right) \\
+\eta \sum_{i} \rho_{i} \Delta x_{t-i}\end{array}$} \\
\hline \multicolumn{5}{|c|}{$\begin{array}{l}\Delta P_{t}=-\alpha+(1-\eta) \Delta x_{t}+\eta \Delta x_{t-1}-\phi y_{c, t}+\left(1-\beta_{4}\right)\left(R E A L * \Delta x_{t}\right) \\
+\beta_{4}\left(R E A L * \Delta x_{t-1}\right)+\left(1-\beta_{5}\right)\left(I T * \Delta x_{t}\right)+\beta_{5}\left(I T * \Delta x_{t-1}\right) \\
+\eta \sum_{i} \rho_{i}\left(\Delta x_{t-i}-\Delta x_{t-i-1}\right)\end{array}$} \\
\hline
\end{tabular}

Considerando-se apenas o período de 1994 a 2002 os resultados mudam ao se estimar por MQI pois o regime de metas de inflação implica em ganhos de 
credibilidade para a política monetária (tabela 8), o que não havia sido observado nas estimações dos períodos anteriores, ou seja, os agentes econômicos dispuseramse a enrijecer suas relações econômicas confiando que o Banco Central estará menos disposto a promover um choque monetário a partir da adoção do regime de metas de inflação.

Tabela 8

Estimações do sistema (3)-(4) por MQI: 1994-2002

\begin{tabular}{lcccc}
\hline & Coeficiente & Erro Padrão & Estatística $t$ & Prob. \\
\hline$\delta \eta$ & -0.0043 & 0.0036 & -1.1976 & 0.2326 \\
$\eta$ & 0.2360 & 0.0649 & 3.6374 & 0.0004 \\
$\phi$ & 0.1321 & 0.1007 & 1.3111 & 0.1915 \\
$\beta_{5}$ & 0.3330 & 0.0962 & 3.4597 & 0.0007 \\
$\rho_{1}$ & -0.4032 & 0.2126 & -1.8967 & 0.0594 \\
$\rho_{2}$ & -0.2143 & 0.1831 & -1.1708 & 0.2432 \\
$\rho_{10}$ & 0.3667 & 0.1880 & 1.9503 & 0.0527 \\
$\rho_{11}$ & 0.8125 & 0.2914 & 2.7878 & 0.0059 \\
$\rho_{12}$ & -0.7378 & 0.2712 & -2.7200 & 0.0072 \\
$\alpha$ & -0.0078 & 0.0032 & -2.4072 & 0.0171 \\
\multicolumn{5}{c}{} \\
\hline$y_{c, t}=-\eta \delta+\eta \Delta x_{t}+\phi y_{c, t-1}+\beta_{5}\left(I T * \Delta x_{t}\right)+\eta \sum_{i} \rho_{i} \Delta x_{t-i}$ \\
$\Delta P_{t}=-\alpha+(1-\eta) \Delta x_{t}+\eta \Delta x_{t-1}-\phi y_{c, t}+\left(1-\beta_{5}\right)\left(I T * \Delta x_{t}\right)$ \\
$+\beta_{5}\left(I T * \Delta x_{t-1}\right)+\eta \sum_{i} \rho_{i}\left(\Delta x_{t-i}-\Delta x_{t-i-1}\right)$ \\
\hline
\end{tabular}

Por outro lado, a correção do problema de simultaneidade nessas estimações leva ao resultado de que o regime de metas de inflação não elevou a credibilidade da política monetária se considerarmos um nível de significância de até $5 \%$ (tabela 9). Em outras palavras, a não-correção do problema de simultaneidade tende a superestimar a credibilidade da política monetária. Tal relação se torna mais contundente a partir do fato de que a simples condução de um teste de exogeneidade (tabela 11), deixa claro que mesmo a hipótese de exogeneidade fraca é significantemente rejeitada, ou seja, o problema de simultaneidade do sistema parece ser inconteste. 
Tabela 9

Estimações do sistema (3)-(4) por FIML: 1994-2002

\begin{tabular}{lcccc}
\hline & Coeficiente & Erro Padrão & Estatística $z$ & Prob. \\
\hline \multicolumn{5}{c}{} \\
$\delta \eta$ & -0.0040 & 0.0030 & -1.3484 & 0.1775 \\
$\eta$ & 0.2838 & 0.0690 & 4.1111 & 0.0000 \\
$\phi$ & 0.2960 & 0.1042 & 2.8397 & 0.0045 \\
$\beta_{5}$ & 0.1796 & 0.0994 & 1.8058 & 0.0710 \\
$\rho_{1}$ & -0.0082 & 0.2243 & -0.0364 & 0.9710 \\
$\rho_{2}$ & -0.0758 & 0.2050 & -0.3698 & 0.7116 \\
$\rho_{10}$ & 0.3075 & 0.1665 & 1.8464 & 0.0648 \\
$\rho_{11}$ & 0.5026 & 0.2123 & 2.3676 & 0.0179 \\
$\rho_{12}$ & -0.5919 & 0.2053 & -2.8832 & 0.0039 \\
$\alpha$ & -0.0077 & 0.0049 & -1.5572 & 0.1194 \\
\multicolumn{5}{c}{} \\
\hline$y_{c, t}=-\eta \delta+\eta \Delta x_{t}+\phi y_{c, t-1}+\beta_{5}\left(I T * \Delta x_{t}\right)+\eta \sum_{i} \rho_{i} \Delta x_{t-i}$ \\
$\Delta P_{t}=-\alpha+(1-\eta) \Delta x_{t}+\eta \Delta x_{t-1}-\phi y_{c, t}+\left(1-\beta_{5}\right)\left(I T * \Delta x_{t}\right)$ \\
$+\beta_{5}\left(I T * \Delta x_{t-1}\right)+\eta \sum_{i} \rho_{i}\left(\Delta x_{t-i}-\Delta x_{t-i-1}\right)$ \\
\hline
\end{tabular}

Tabela 10

Testes de Wald - $H_{0}: \beta_{4}=\beta_{5}$

\begin{tabular}{lccc}
\hline Estimador & Período & Estatística $\chi^{2}$ & Prob. \\
\hline MQO & $1980-2002$ & 1.4107 & 0.2349 \\
MQI & $1980-2002$ & 0.1079 & 0.7425 \\
FIML & $1980-2002$ & 1.8497 & 0.1738 \\
MQI & $1990-2002$ & 0.1348 & 0.7135 \\
FIML & $1990-2002$ & 0.1281 & 0.7204 \\
\hline
\end{tabular}

Tabela 11

Teste de exogeneidade de Hausman, $H_{0}: \Delta x_{t}$ é exógena

\begin{tabular}{cc}
\hline Estatística & Prob. \\
\hline 5.2219 & 0.0000 \\
\hline
\end{tabular}

Tabela 12

Teste de Garbade, $H_{0}: \mathrm{Q}=0$

\begin{tabular}{cc}
\hline Estatística & Prob. \\
\hline 161413.5 & 0.0000 \\
\hline
\end{tabular}

Assim, a questão que passa a ser levantada é: uma vez que chegamos a resultados tão distintos a partir de amostras diferentes, a que conclusão podemos chegar sobre as variações de credibilidade promovidas pela adoção do regime de 
metas de inflação? A fim de lançar luz sobre a resposta a tal questão o próximo tópico visa construir um índice mensal para a credibilidade da política monetária, nos fornecendo uma visão mais precisa das variações temporais da credibilidade ocorridas no Brasil.

\subsection{Um índice de credibilidade para o Brasil}

A análise anterior mostrou que o coeficiente que visa fornecer uma medida plausível para credibilidade, não se mantém constante ao longo do tempo. Portanto, foi possível observar, por exemplo, que mudanças de políticas implicam diretamente em alterações do parâmetro de credibilidade, como já esperado, dada a consideração de Lucas (1973) sobre o modelo aqui pré-disposto, e a consideração de Faust e Svensson (1998) sobre o comportamento temporal da credibilidade da política monetária.

Logo, objetiva-se nessa seção observar as variações do parâmetro de credibilidade ao longo do tempo, sem impor de forma prévia crenças pessoais sobre os pontos de quebra estrutural como realizado na seção anterior, onde os pontos de quebra para o Plano Real e para a adoção do regime de metas de inflação foram pré-estabelecidos à análise. Para tanto, utiliza-se um modelo de estado-espaço pela condução do filtro de Kalman para o parâmetro de credibilidade, seguindo a metodologia proposta por Hardouvelis e Barnhart (1989), configurando-se um modelo onde as equações de medida seguem as definidas na seção teórica e a equação de transição é dada por:

$$
\eta_{t}=\eta_{t-1}+v_{t}
$$

onde $u_{i t} \sim N\left(0, \sigma^{2}\right), i=1,2$ e $v_{t} \sim N\left(0, Q \sigma^{2}\right)$. A da forma funcional de (5) deve-se primariamente a sua simplicidade, uma vez que esta análise não visa a construção de um processo estocástico para a geração dos $\eta^{\prime}$ s, mas testar a estabilidade de $\eta_{t}$, bem como obter um padrão para a sua variação temporal. ${ }^{7}$ Os termos de erro das equações de medida e da equação de transição são mutuamente e serialmente não correlacionados. O parâmetro $Q$ que multiplica a variância $\sigma^{2}$ construindo a variância de $\eta$ é o parâmetro a ser testado para concluir-se sobre a possível

\footnotetext{
${ }^{7}$ De fato, até onde sabemos, não há uma forma funcional definida para o índice de credibilidade como estabelecido neste estudo na literatura. A forma funcional de (5) está de acordo, porém, com premissas de evolução da credibilidade da política monetária sob outras formas de medir credibilidade, como em Hardouvelis e Barnhart (1989). Além disso, diversas outras formas funcionais foram utilizadas não se alterando significativamente os resultados
} 
estabilidade de $\eta$, onde se $Q=0, \eta$ pode ser considerado constante, e o coeficiente da regressão remete-se ao coeficiente linear estimado comumente.

Garbade (1977) descreve a estimação do modelo. Sob tal aspecto, as doze observações iniciais obtidas do ano de 1980 são utilizadas para inicializar o filtro de Kalman. Assim, iniciando-se no ano de 1981, o filtro de Kalman atualiza seqüencialmente a estimação do coeficiente e a matriz de covariância pelo próximo período. Utilizando o procedimento de uma estimação de máxima verossimilhança em conjunto à estimação do modelo de medida, obtém-se uma estimação para $Q$, podendo-se verificar a estabilidade de $\eta$. Tal teste de variabilidade temporal do parâmetro é chamado de teste de Garbade. Sob tal contexto, as estimações para a amostra considerada e a aplicação do teste de Garbade rejeita a hipótese de $Q=0$, ou seja, indica-se a existência de variabilidade temporal de $\eta$ (tabela 12).

Sendo realizada a estimação do modelo nós obtemos uma série estimada para $\eta$, ou seja, uma série estimada para a credibilidade da política monetária. Tal série é disposta nas figuras 1 e 2, onde a figura 1 apresenta a série completa com o seu intervalo de confiança e a figura 2 a série a partir da implementação do Plano Real.

Figura 1

Evolução da credibilidade da política monetária

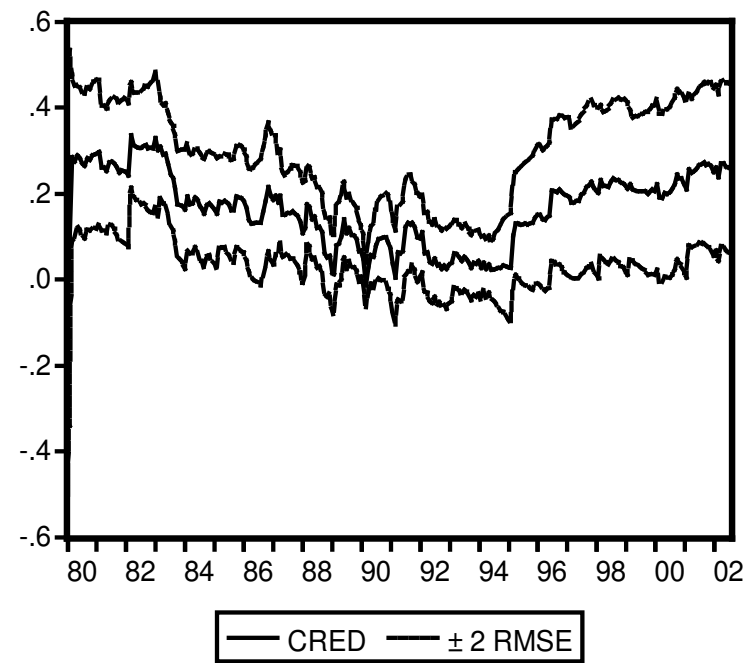


Figura 2

Evolução da credibilidade da política monetária - pós-Real

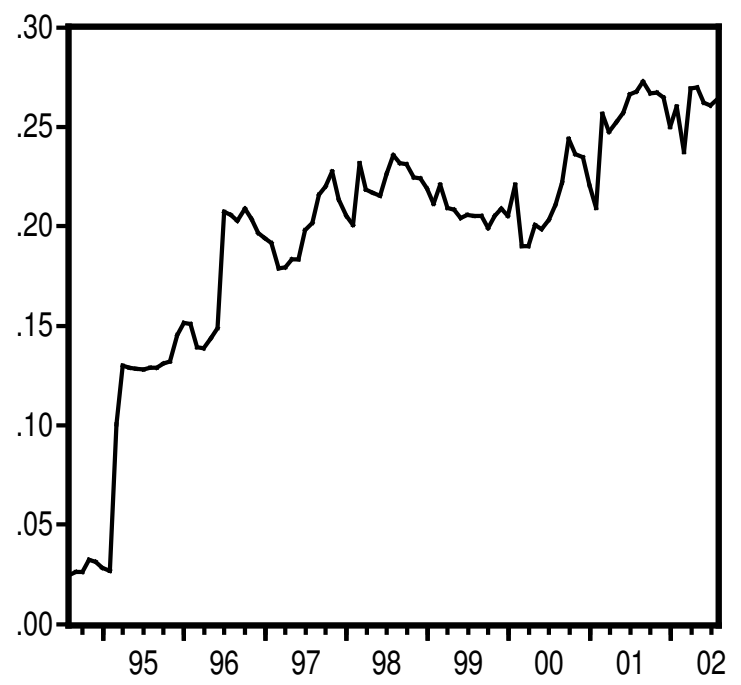

Ao analisar a figura 1 é possível observar que a década de 80 foi marcada por uma tendência de queda da credibilidade da política monetária sem precedentes, tendo continuidade no início da década de 90, passando de patamares próximos de 0.4, para valores próximos de zero. Além disso, a conclusão de Svensson (1993) de que após a implementação de políticas monetárias alternativas, ocorre uma queda imediata de credibilidade, seguida de posterior recuperação é comprovada para o Brasil, tendo tal fenômeno ocorrido notadamente em três ocasiões: O Plano Cruzado (em 1986), o Plano Real (em 1994) e a adoção de Metas de Inflação (em 1999).

Com relação à figura 2, nós podemos alcançar um resultado esclarecedor sobre a possível controvérsia lançada na seção 3.1. Nesse sentido, a queda imediata de credibilidade após o Plano Real parece ter impactado negativamente sobre a média do período, de forma que o efeito positivo das metas de inflação sobre a credibilidade parece ser superestimado se considerarmos esse período imediatamente após a implementação do Real na análise comparativa. 
Ao se considerar especificamente o período pós implementação de metas de inflação é possível notar que ocorre uma pequena queda em um primeiro momento, que alcança a recuperação no momento seguinte. Isso corrobora com a hipótese explicitada por Svensson (1993) e explica em parte as estimações da sub-seção anterior onde o regime de metas de inflação não teria elevado o nível de credibilidade da política monetária.

Tais resultados corroboram com os obtidos por Minella et alii (2003) onde fica evidenciado que a autoridade monetária no Brasil tem reagido fortemente a expectativas de inflação para manter a inflação estável e a sua credibilidade econômica, o que é vital para manter a eficiencia do regime de metas de inflação. Em outras palavras que o Banco Central tem evitado a utilização do trade-off inflação-produto para elevar o produto de curto-prazo, dando prioridade total ao controle da inflação. Isso significa um processo importante de construção de credibilidade.

Tal aspecto também é ressaltado por Silva e Portugal (2002) que indicam a partir de uma função de reação do Banco Central a là Taylor que a trajetória da credibilidade da política monetária a partir da adoção do regime de metas de inflação segue um padrão de "credibility construction", com um aumento do conservadorismo do Banco Central.

Ao mesmo tempo, é possível observar que o período pode ser dividido em dois momentos distintos. O primeiro, antes do Plano Real, onde a credibilidade apresentou uma tendência continuamente descendente, e o segundo, após o Plano Real, onde a credibilidade da política monetária apresentou uma tendência nitidamente crescente. Nesse período pós-Real é possível observar a construção da credibilidade da política monetária em um processo contínuo, onde uma característica marcante é a capacidade da credibilidade não ser significantemente abalada pelas seguidas crises ocorridas no período. Nesse sentido, a política monetária no período apresentou uma reação contracionista extremamente forte a tais crises de forma a evitar-se um abalo sob a credibilidade.

Nesse sentido, o tamanho limitado da série de dados após a adoção do regime de metas de inflação parece impedir uma conclusão mais contundente sobre a relação entre credibilidade e o regime de metas de inflação. Entretanto, as evidências apresentadas parecem ser suficientes para apontar favoravelmente para os efeitos da adoção das metas de inflação sobre a credibilidade da política monetária. 


\section{Conclusões e Implicações Políticas}

A principal contribuição do presente artigo é a avaliação dos possíveis ganhos de credibilidade da política monetária a partir da implementação do regime de metas de inflação. Nesse sentido, considera-se que a credibilidade da política monetária é obtida pela não exploração do trade-off entre produto e inflação, seguindo assim Lucas (1973), Faust e Svensson (1998) e Razzak (2001).

A análise é conduzida em duas partes complementares. A primeira constitui da estimação da credibilidade da política monetária para o período 1980-2002 com dados mensais, focalizando com atenção primária as variações de credibilidade ocorridas por intermédio do Plano Real e da adoção do regime de metas de inflação. Os resultados distanciam-se de uma relação óbvia, uma vez que, embora os ganhos de credibilidade ocorridos mediante o Plano Real sejam incontestáveis, os ganhos de credibilidade advindos da da adoção do regime de metas de inflação não são observados em todas as estimações realizadas.

A fim de se lançar luz sobre tal dilema a segunda parte da análise buscou construir um indicador mensal de credibilidade de política monetária a base de um modelo de estado-espaço, por meio da condução do filtro de Kalman ao parâmetro de credibilidade. A construção de tal indicador visa fornecer uma ferramenta útil para a análise das reações da credibilidade dos agentes aos mais diversos choques exógenos ao modelo, embora essa não seja a finalidade central do presente estudo.

No que se refere a observação das mudanças do indicador de credibilidade a partir da adoção do regime de metas de inflação, foi possível observar uma queda inicial da credibilidade da política monetária corroborando com Svensson (1993), que sugere que os ganhos de credibilidade a partir de metas de inflação estão sujeitos a uma defasagem. Tal período de ajuste das expectativas é apontado como o provável causador dos resultados conflitantes da parte inicial da análise. A partir de então, tendo a economia passado pelo período de ajuste, a credibilidade da política monetária assume uma trajetória levemente crescente, indicando uma direção favorável à idéia de que o regime de metas de inflação afeta favoravelmente a credibilidade.

Assim, embora o tamanho limitado das séries sob a qual o Brasil está sujeito ao regime de metas de inflação não possibilite uma afirmação contundente sobre os possíveis ganhos de credibilidade da adoção de tal regime, as evidências apresentadas parecem apontar favoravelmente à relação entre metas de inflação e credibilidade da política monetária. 


\section{Referências}

Ball, L. (1994). Credible disinflation with staggered price setting. American Economic Review, pages 282-289.

Ball, L. (1995). Disinflation with imperfect credibility. Journal of Monetary Economics, pages 5-23.

Ball, L., Mankiw, G., \& Romer, D. (1988). The New Keynesian economics and the output-inflation trade-off. Brookings Papers on Economic Activity, 1.

Blanchard, O. (1984). The Lucas critique and the Volcker deflation. Working Paper 1326.

Blinder, A. S. (1999). Central Bank credibility: Why do we care? How do we build it? NBER Working Paper 7161.

Calvo, G. (1983). Staggered prices in a utility prices in a utility maximizing framework. Journal of Monetary Economics, 12:383-398.

Cukierman, A. e Meltzer, A. (1986). A theory of ambiguity, and inflation under discretion and asymmetric information. Econometrica, 54:1099-1128.

Faust, J. \& Svensson, L. (1998). Transparency and credibility: Monetary policy with unobservable goals. NBER Working Paper 6452.

Federal Reserve Bank of Kansas City (1996). Achieving price stability. Kansas City, MO.

Friedman, M. (1968). The role of monetary policy. American Economic Review, pages $1-17$.

Garbade, K. (1977). Two methods for examining the stability of regression coefitients. Journal of the American Statistical Association, 72:54-63.

Hardouvelis, A. (1985). Exchange reates, interest rates amd stocks announcements: A theoretical exposition. Journal of International Money and Finance, 4(4):44354.

Hardouvelis, A. \& Barnhart, W. A. (1989). The evolution of federal reserve credibility: 1978-1984. The Review of Economics and Statistics, 71(3):385-393.

Lucas, R. (1972). Expectations and the neutrality of money. Journal of Economic Theory, 4:103-124. 
Lucas, R. (1973). Some international evidence on output-inflation trade-offs. The American Economic Review, 63(3):26-334.

Mankiw, G. (1985). Small menu costs and large business cycles: A macroeconomic model of monopoly. Quarterly Journal of Economics, 100:529-539.

Minella, A., Freitas, P. S., Goldfajn, I., \& Muinhos, M. K. (2003). Inflation targeting in brazil: Constructing credibility under exchange rate volatility. Journal of International Money and Finance, 22:1015-1040.

Perry, G. (1983). What have we learned from disinflation? Brookings Papers on Economic Activity, 2:587-602.

Phelps, E. (1968). Money-wage dynamics and labour market equilibrium. Journal of Political Economy, 76:678-711.

Razzak, W. A. (2001). Are inflation-targeting regimes credible? Econometric evidence. Reserve bank of New Zealand Working Paper.

Rotemberg, J. (1982). Monopolistic price adjustment and aggregate output. Review of Economic Studies, 44:517-531.

Sargent, T. (1982). The ends of four big inflations. In Hall, R., editor, Inflation: Causes and Effects. University of Chicago Press, Chicago.

Sargent, T. (1983). Stopping moderate inflations: The methods of Poincare and Thatcher. In Dornbusch, R. \& Simonsen, M. H., editors, Inflation, Debt and Indexation. MIT Press, Cambridge, Massachusetts.

Silva, M. \& Portugal, M. (2002). Inflation targeting in Brazil: An empirical evaluation. XXX Encontro Nacional da ANPEC, Nova Friburgo-RJ, Brasil.

Svensson, L. (1993). The simplest test of inflation target credibility. NBER Working Paper Series, n.4604.

Taylor, T. (1980). Aggregate dynamics and staggered contracts. Journal of Political Economy, 88:1-23. 